function. Hence the work of the damaged kidneys is diminished and the dangerous complications which are associated with renal disease are delayed. Again, in chronic cardiac disease the lowered function and diminished blood-pressure which attend on life in a relaxing climate relieve the strain on the cardiac muscle, and so favour its possibilities of endurance. In the treatment of the degenerative processes which affect the central nervous system the relaxing climate is the only safe medium. The vital forces have to be conserved so that the unfavourable movement of the morbid process may be checked. In neurasthenia and hysteria the therapeutic problem, so far as climate is concerned, is less simple. Temperamental considerations and local circumstances must be borne in mind, as also the mental attitude and reaction of the patient. The possibilities or otherwise in reference to suitable baths and other mechanical methods of treatment are also guiding influences in the selection of a suitable climatic station, and this more particularly in cases of chronic renal and chronic cardiac disease.

$$
1 \text { Edin. Med. Jour., March } 1905 .
$$

\section{THE TREATMENT OF LOCOMOTOR}

\section{ATAXY.}

IT is hard to exaggerate the extent to which success in the treatment of grave, chronic organic disorders depends upon the mental attitude adopted by the practitioner in charge. Any patient who knows himself to be the victim of such a malady is sure to be oppressed, to some degree, with the sense of his unfortunate position, and the oppression is easily aggravated by pessimism in his medical attendant. It is easy for a doctor called upon to treat such an inveterate disease as locomotor ataxy to yield to a conviction of the hopelessness of cure, and to assume an attitude of what may be called vicarious stoicism. This, of course, is the worst thing possible for his patient, for it merely serves to indulge the melancholy outlook natural to a sick man. For this reason they cannot be sufficiently commended who set themselves to a determined attempt to achieve the maximum of alleviation possible under the circumstances, and the extent to which such alleviation may be carried is not seldom matter for gratified surprise. The mere fact that a skilled observer will undertake radical measures for his betterment is by itself sufficient, by pure suggestion, to lay the foundation in a patient for all the minor improvements in bodily health and spirits which, without pretending to be called cure, yet go far to make an afflicted existence tolerable and useful, if not actually a pleasure.

Dr. Hinsdale ${ }^{1}$ has recently published what he calls a "hopeful case of locomotor ataxy," the details of which are as follows:-A man, aged 42, who denied that he had had syphilis, was none the less a typical example of locomotor ataxy. He suffered greatly from gastric crises and lightning pains, and was reduced to a miserable state of health. He was put to bed and underwent a "rest-cure," that is, rest, massage, and high feeding, with the addition of "sinusoidal" electricity, and the educational movements of the extremities which have for some time been practised in the treatment of this affection. In the matter of medication it was found that he was very intolerant of iodides, so he was treated with nitrate of silver and belladonna internally, and with antipyrin for the relief of the pains. In the course of three months he put on $38 \mathrm{lbs}$. of weight, the pains and ataxy vastly improved, and he was enabled to return to his usual occupation. Three years later he reappeared in a relapsed condition, his gastric crises being particularly severe. He was again submitted to similar treatment, with the addition of $\frac{1}{20}$ th of a grain of mercuric chloride given under the skin twice daily. At the date of this treatment he was greatly bothered by inefficiency of his rectal sphincter, for the relief of which faradism was daily applied to the rectum. In nine weeks he gained 46 pounds in weight, and was sufficiently recovered in all respects to resume his occupation, although the sphincter trouble had not improved in proportion to the other symptoms.

Dr. J. Monroe Lieberman, ${ }^{2}$ again, publishes some extraordinarily good results which he claims to have gained by exposing the region of the lumbar cord to the action of ultra-violet rays, results so good as to prompt scepticism. We do not propose to set out the details of the method, which may be found according to the above reference, but the routine, in the author's own words, is as follows :- (1) A warm half-bath at night before going to bed, with light massage; (2) ultra-violet rays in sittings of 10-30 minutes, thrice weekly ; (3) static electricity by means of the Morton wave current or wooden brush, 15 to 20 minutes daily.

1 Jour. of Amer. Med. Assoc., Feb. 18, 1905. 2 New York Med, Jour., Feb. 18, 1905.

\section{THE TREATMENT OF INCIPIENT INSANITY.}

CUstom is a good servant but a bad master, and its power is nowhere more in evidence than in the hidebound attitude both of the public and the law towards the psychoses. To them the affections of the mind, mysterious as we must acknowledge them to be, are so mysterious as to be beyond the application of considerations adapted to more corporeal maladies. Otherwise, as was pointed out by Sir John Batty Tuke before the Neurological Society, there would not exist, as unfortunately there does, a disinclination to afford the insanities the early treatment which is recognised as the essential of success in all cther forms of disease. At present no case of insanity, except among the moneyed classes, can be submitted to really suitable treatment until the condition is sufficiently far advanced to warrant certification ; until, that is to say, the most invaluable epoch of the disease has been allowed to slip away. What is required, said the speaker, is, in the first place, an increase in the number of Lunacy Commissioners, and in the second place institutions for the reception of early cases of mental disease among the poor. He quoted the state of affairs that obtains in Germany. In that country there are attached to each of the 20 universities clinics of psychiatry under the superintendence of a professor. Special accommodation is provided, and through these clinics pass all persons reputed to be of unsound mind. Inveterate cases are handed on to the asylums, but all slighter and more hopeful cases are treated to a termination in these clinics - cases, for instance, such as neurasthenia, or insanity following 
fevers or operations. These are not reported to the State Office at all.

It appears that in this country Glasgow has the credit for the first departure in this direction, and the experiment has been thoroughly successful. It remains for the profession to educate public opinion in the knowledge that insanity, like other diseases, is capable of rational treatment; that the first essential of all successful treatment is its application before the condition to be dealt with has become inveterate; and that it is better economy to preven's an establishment of insanity, and to retain a good citizen, than to make adequate provision for a useless member of the community after sufficient pains have been taken to render him permanently useless.

\section{THE ETIOLOGY OF CARCINOMA.}

Dr. Beatson, in a paper read before the MedicoChirurgical Society of Edinburgh, on March 1st, supported the germinal theory of carcinoma which led him, nine years ago, to suggest the removal of the ovaries in mammary cancer. The writer referred to the recent work of Doyen and that of Ford Robertson and Wade, but held that neither of these observers had fulfilled Koch's postulates in the absence of successful inoculation experiments. Dr. Beatson maintains that removal of the ovaries in patients suffering from mammary cancer has proved of benefit in 36 per cent. of all cases so treated. After oophorectomy the cancer cells degenerate while the stroma increases, the tumour undergoing changes which may lead to its entire disappearance. The changes of the nature of reducing division seen in cancer cells are very similar to those observed by Farmer during the development of normal repro. ductive tissue. These facts strongly. suggest a germinal origin for carcinoma. The almost exclusively cellular character of a cancerous tumour without any attempt at organisation is difficult to reconcile with a microbic origin, as also the absence of signs of irritation when cancer cells find their way into a distant organ. Neither in primary nor in secondary malignant tumours do symptoms arise till the growth has attained sufficient size to act as a foreign body. The delay of patients in applying for advice is related to this, and is to a great extent unavoidable.

The presence of cancer is frequently associated with a change in the body fat which is of a peculiar reddish-yellow colour, and has been ascertained to contain lutein. Whether this change is peculiar to cancer is not known, but the colour of the fat may give rise to the yellow complexion which is characteristic of advanced cases of the disease.

A further analogy between a cancerous tumour and the reproductive organs is to be seen in their relations to the storage of fat by the body, their activity leading to its diminution, while their degeneration or removal favours its accumulation. It may be that the cancer cells perform some physiological part in the elimination of some product of the body or vicariously assist in some function of the reproductive glands. In this connection the nitrifying nodules on the roots of bean plants are of great interest. Should the germinal theory of cancer prove the true solution of the problem there is no reason to despair of successful treatment.

\section{THE INFANT AT BIRTH.}

Is a lecture given at the Institute of Hygiene last week on the Physiology, Care, and Management of the Infant at Birth, Dr. W. G. McDowell referred to the paucity of literature bearing on the subject and impressed upon his hearers the necessity of using to the best advantage such practical information as is available in order to preserve the infant from the evil influences that surround him at the present time. It is not generally allowed, he said, that most babies are born perfectly healthy, yet this is so, for Nature has not only a wonderful way of pinching out an undesirable strain, but also a tendency to adjustment in all her processes. Those who enter life burdened with hereditary taint, and those who are physically or mentally decrepit at birth are, after all, comparatively few. What then is the cause of so much mortality during the first years of life, when no less than about 15 per cent. of those born cease to exist? The mother has, crystallised into instinct, the experience of ages to guide her. Why should she err in the bringing up of her offspring? But she does, and it is this fact which calls for the admonition to go back to nature.

The child's physiological conditions should bestudied and his requirements learned. The imperfect development of the brain, till the end of the seventh year, is a fact that should prevent a child from being sent to school at an early age. The children of the poorer classes are, oftener than not, sent to school at the age of three, when they should be living the freest pos. sible outdoor life. A child's brain loves activity, but it must work in its own way - not at the behest of taskmasters who fix his attention for long periods and try to compel steady application. Dr. Jacobi has maintained that no child should commence school studies till it is nine years of age, and there is a good deal to be said in favour of his contention.

In speaking of the special senses at birth, Dr. $\mathrm{McD}$ owell pointed out that sight and hearing are defective to a degree that might greatly surprise the mother if she were allowed to find it out for herself in the case of her own young babe. The infant is able to detect the presence of a strong light and to differentiate between light and darkness but it cannot see its mother's face for some weeks. The recognition of colours comes only after a few months. He first distinguishes yellow, red, pure white, gray, and black, while green and blue come later. Taste is evident at birth, but hearing is not sufficiently developed for him to hear much more than his own cry.

One of the strongest points insisted upon was, of course, the avoidance of adventitious feeding soon after birth, and the advisability of depending upon the food provided by nature which is the only food physiologically fit for consumption by the new-born infant. Nature actually goes out of her way to make a special kind of milk for the infant's first few meals to suit his temporary needs, but it is an all too common thing to find Nature forestalled by ignorance and the child dosed with a confection of traditional veneration and misplaced respect-butter and sugar.

The final remark of the lecturer was to the effect that those who disregard the ways of Natare in bringing up their children, go out to meet sorrow and generally have not far to go. 